\title{
Cross-cultural analysis of medicinal plant use practice in four (Gurage, Mareqo, Qebena and Silti) ethnic communities in south central Ethiopia
}

Alemtshay Teka ( $\sim$ alemtshay.teka27@gmail.com )

Kotebe Metropolitan University https://orcid.org/0000-0002-3634-7650

\section{Zemede Asfaw}

Addis Ababa University College of Natural Sciences

Sebsebe Demissew

Addis Ababa University College of Natural Sciences

Patrick Van Damme

Ghent Univeristy

\section{Research}

Keywords: Culture, ethnic groups, Gurage, Mareqo, medicinal plants, traditional knowledge, Qebena, Silti

Posted Date: January 8th, 2020

DOI: https://doi.org/10.21203/rs.2.20408/v1

License: (c) This work is licensed under a Creative Commons Attribution 4.0 International License.

Read Full License 


\section{Abstract}

Background: Ethnic groups throughout the world have developed their own cultures expressed in the form of customs, taboos and traditional healthcare systems. Traditional medicine system is one of the widespread cultures known throughout the world which is very much tied to cultural practices of the community or ethnic group. Medicinal plant treasure found in Gurage and Silti Zones remained poorly characterized and understood. Therefore, this study was conducted in four ethnic groups (viz. Gurage, Qebena, Mareqo and Silti) which have lived in close proximity and contact for many centuries in the two Zones. In the present study unique and shared cultural elements in connection to traditional herbal medicine were examined through investigation of the diversity of medicinal plants. Moreover, attempts have been made to determine similarities among the society in the medicinal plants they have used in general and in medicinal plant species considered culturally most important.

Methods: In a study that involved 320 randomly samples informants semi-structured interviews, focus group discussions, participant observation were used and qualitative and quantitative data were collected. Descriptive statistics, rank order priority (ROP), Jaccard similarity coefficient and clustering were used for data analysis.

Results: A total of 244 medicinal plant species and a fungal species used to treat human and/or livestock ailments were documented. The number of plants (80 plants, $33 \%$ ) with ROP value greater than $50 \%$ is considerably smaller than that of plants with ROP $<50 \%(164,67 \%)$. Jaccard similarity index and clustering analysis for all cited plants, among the respective studied districts, indicated that grouping generally followed the existing ethnic origin. On the contrary, clustering based on culturally important medicinal plant species ( 80 plant species, score ROP $\geq 50 \%$ ) showed the influence of proximity and geographical orientation rather than ethnic relation.

Conclusions: Culturally most important plants ( $80 \mathrm{spp}$.) are widely used and best shared with nearby communities and this could imply current (new) knowledge being practiced in the community. This knowledge must be documented and better utilized in a modern way including modernized use of traditional medicinal plants.

\section{Introduction}

Ethnic groups throughout the world have developed their own cultures expressed in the form of customs, taboos, songs, traditional foods and healthcare systems. Traditional medicine selected based on several thousands of years of experience has been major aspect of cultural heritage [1]. Traditional medicine system is one of the widespread parts of culture known throughout the world [2]. Like other kinds of local knowledge, it is very much tied to cultural practices of the community or ethnic group $[3,4]$. Its role in the healthcare system is enormous and widely recognized. The word 'culture' refers to the characteristics and knowledge of a particular group of people, defined by everything from language, religion, cuisine, social 
habits, song, story and arts [5]. In the present study, the type of plants used in a group of people in their traditional health care system is considered as part of culture.

Ethnomedicinal knowledge, which develops from the interaction of a given culture with the local biophysical environment or locally available plants, is diverse and sometimes it could be ecosystem and ethnic community specific [6, 7]. Factors such as social, ecological, cultural background (incl. religious, linguistic) and ancestral inheritance determine the kind of traditional herbal knowledge developed in a community. Owing to these facts, herbal knowledge varies hugely across different community, geographic setting or ethnic groups [7-9]. The influence of cultural background and ancestral inheritance could be seen in the variation of people's perceptions and plant use preference in a community inhabiting same geographical or ecological area, facing similar environmental factors $[9,10]$. As a result, comparative studies in traditional knowledge and plant use culture between communities or ethnic groups gives an opportunity to investigate how the local flora is understood and used in daily life, health practices and, ultimately for survival under different cultural settings [11].

Various studies conducted in different corner of the world have shown the variation that exist in plant use knowledge and ethnomedicinal healing systems across cultures and agro-ecology [12-16]. Traditional medicinal plants use could also vary among communities within the same ethnic group [17] and geographic area [18]. Thus, medicinal plant use practice can be considered to show the cultural differences that may possibly exist between traditional societies or ethnic groups inhabiting in similar/different geographic locations.

Recently, the knowledge of traditional peoples and accompanied systems is disappearing at increasing rate. Besides medicinal plant treasure found in Gurage and Silti Zones, is not fully explored and hence it remained poorly characterized and understood. In light of these scenarios the present study is conducted to document the traditional medicinal plants known in the societies of the two zones. The study area harbor four ethnic groups (viz. Gurage, Qebena, Mareqo and Silti) which have lived in close proximity and contact for many centuries. In the present study unique and shared cultural elements in connection to traditional herbal medicine were examined through investigation of the diversity of medicinal plants. Moreover, attempts have been made to determine similarities among the society in the medicinal plants they have used in general and in medicinal plant species considered culturally most important.

\section{Methods}

\section{Study area}

Gurage and Silti zones are situated in Southern Nations, Nationalities and Peoples Region (SNNPR) and located in south central Ethiopia. Gurage Zone is located at $7^{\circ} 40^{\prime} 0^{\prime \prime}-8^{\circ} 30^{\prime} 0^{\prime \prime} \mathrm{N}$ and $37^{\circ} 50^{\prime} 0^{\prime \prime}-38^{\circ} 40^{\prime} 0^{\prime \prime}$ E with altitudinal range stretching between $1000-3600 \mathrm{~m}$ a.s.l. and covers an area of $5893.5 \mathrm{~km}^{2}$ [19]. On the other hand, Silti Zone is located at $7^{\circ} 40^{\prime} 0^{\prime \prime}-8^{\circ} 10^{\prime} 0^{\prime \prime} \mathrm{N}$ and $37^{\circ} 50^{\prime} 0^{\prime \prime}-38^{\circ} 40^{\prime} 0^{\prime \prime} \mathrm{E}$ with altitudinal range 
stretching between 1640 - $3277 \mathrm{~m}$ a.s.l. and cover an area of $2537.5 \mathrm{~km}^{2}$. The geographical locations of the two zones and the studied districts are shown in Figure 1.

Gurage Zone consists of peoples belonging to the Gurage, Qebena and Mareqo ethnic groups. These ethnic groups speak Guragigna, Qebena and Libido languages respectively. Cheha, Meskan, Muhir-Aklil and Sodo districts of the study area represent the Gurage ethnic group. Silti Zone is comprised of people identified as the Silti ethnic group. These people speak Siltigna and live in Silti and Wulbareg districts. The geographic proximity between Gurage and Silti zones led to extensive intermarriage between the four ethnic groups (Gurage, Silti, Mareqo, Qebena) and maintain inter-ethnic contacts [20]. This mutual influence has been shown in the respective ways of living which most likely resulted from this cultural mix [20]. Extensive cultivation of ENSET (Ensete ventricosum), traditional housing, artifacts, and mode of production are the best-known shared cultural practices of the zones and at large in SNNPR [21]. ENSET is the main food crop together with Hordeum vulgare (barley), pulses, potatoes, and cabbage. The major cash crops are Catha edulis (CHAT), Coffea arabica (BUNA), Eragrostis tef (TEFF) and Guizotia abyssinica (NOUG). Animal husbandry is also part of subsistence farmer's way of life. Amharic language is widely spoken by the communities and sometimes it is used as lingua franca in the zones. Based on the recent classification of potential vegetation types as described in Friis et al. [22], the study area is dominantly characterized by the dry evergreen Afromontane forest and grassland complex (the undifferentiated Afromontane forest subtype) (Figure 2).

\section{Informant selection and Data collection}

A total of 320 informants (40 from each study district) were selected randomly following Gomez-Beloz, [23]. Semi-structured interviews during walk-in-the-woods or by using plant 'props' (freshly collected plant material or photographs), focus group discussions, participant observation and market survey were applied for data collection [24-26]. Ethnobotanical information including list of all medicinal plants and parts used; kind of humans/livestock ailments treated and medicinal plants that are sold in the local market were documented. Interview was conducted in Amharic and local languages. Written permission to conduct the research was obtained from the respective zone and district administrative officials. Prior informed consent was obtained from each informant before every interview.

\section{Plant collection and Identification}

All plant specimens were collected, dried, identified and deposited in National Herbarium (ETH) of Addis Ababa University. Identification was made by using Flora of Ethiopia and Eritrea [27-34], in comparison with authenticated specimens from the herbarium and later confirmed by senior taxonomists of the herbarium.

\section{Data analysis}

Corrected fidelity level (rank order priority) was used to identify the most culturally important medicinal plants from each study districts [35]. Rank order priority (ROP) was calculated using the formula: ROP= 
FL C RPL. Fidelity Level (FL) is used to quantify the percentage of informants confirming the use of a plant species for the same major purpose. It is computed as: $\mathrm{FL}(\%)=I p / I u$ C100

Where $\mathbf{I} \boldsymbol{p}$ refers to the number of informants who indicated a specific medicinal plant species is used to treat the same major ailment, and $\boldsymbol{l} \boldsymbol{u}$ is the total number of informants who mentioned the plant for treating any ailment. A high FL value (near 100\%) for a plant indicates that all of the use-reports mentioned the plant for a specific treatment, regardless of the number of times mentioned. Whereas, a low FL value is obtained for plants that are used for many different purposes, and/or known by few informants. However, plants known by few informants for the treatment of limited number of ailments might have high FL values [36]. In addition, plants with similar FL values but known to different numbers of informants may vary in their healing potential. Therefore, to minimize such kind of inconvenience only plants mentioned by at least three informants were considered. In order to differentiate the healing potential of plants with similar FL value a correlation index called Relative Popularity Level (RPL) was calculated $[35,37]$. RPL has values ranges between 0 and 1 . The range categorize the plants into 'popular' $(\mathrm{RPL}=1)$ and 'unpopular' (RPL $<1)$ groups. Popular plants are cited by more than half of the total number of informants (For example in case of Qebena the highest number of informants that cite a plant for of any therapeutic effect is 30 informants. So, plants cited by 15 or more informants are considered as popular, RPL score equals to 1 . RPL is given less than 1 for 'unpopular' plants which are cited by less than 15 informants). Exact RPL values for 'unpopular' plants was determined by dividing total number of informants who mentioned the plant for treating any ailment to half of the maximum number of informants (lu/15 in case of Qebena). Finally, ROP was calculated by multiplying FL values by RPL values.

Jaccard similarity coefficient was used to compare medicinal plants report similarity between the districts. In order to analyse the variability in medicinal plant species among districts, two presence or absence matrices were created. First matrix considered all medicinal plant species cited by informants and the second matrix considered plant species that score minimum rank order priority of 50\% (ROP $\geq$ $50 \%)$. The similarity between any pair of study site in terms of medicinal plant species mentioned was calculated using the Jaccard similarity coefficient:

\section{See Formula 1 in the Supplemental Files.}

where $\mathrm{Jl}$ is the Jaccard similarity index, 'a' is the number of species shared by or common to any compared pair of study sites, and ' $b$ ' and ' $c$ ' are the number of medicinal plant species reported solely in one study district ( $b$ for one study site and $c$ for the other). JI values range between 0 and 1 , whereby a value of 1 indicates complete similarity. Then the similarity coefficient for each pair of study site was used to obtain a dendrogram using unweighted pair-group method analysis (UPGMA; links a new item to the arithmetic average of a group) [38]. Cluster analysis is generally used to group study sites into categories based on their dissimilarities or partition heterogeneous elements into relatively homogenous groups [39]. 


\section{Results}

\section{Medicinal plants used to treat human ailments}

A total of 213 plant species belonging to 79 families and 175 genera and a fungus species were documented and collected (Additional file 1). In terms of percentage of plant species, the family Asteraceae (25 spp., 12\%) appeared to be the most dominant plant family followed by Lamiaceae (20 spp., 10\%), Fabaceae (11 spp., 5\%), Euphorbiaceae (9 spp., 4\%) and Solanaceae (9 spp., 4\%).

\section{Ethno veterinary medicinal plants diversity}

The medicinal plants used to treat livestock consisted of 95 species, in 82 genera and 48 families (Additional file 2). The most commonly mentioned plant families containing ethnoveterinary species were Asteraceae (11 spp., 12\%), Lamiaceae (8 spp., 9\%), Solanaceae (8 spp., 9\%) and Fabaceae (6 spp., 7\%).

\section{Relative healing potential of medicinal plants: Rank order priority (ROP)}

Relative healing potential of medicinal plants was computed for all reported medicinal plants. Eighty species were identified as the most preferred plants (ROP $\geq 50 \%$ ). The list of most important species $(\mathrm{ROP} \geq 50 \%)$ along with their use categories and reported study district are provided in Additional file 3. Ajuga integrifolia, Clerodendrum myricoides, Hagenia abyssinica, Ruta chalepensis, and Solanum incanum (ROP $=100 \%$ ) had high fidelity level for treating infectious and intestinal parasitic diseases. Diseases of the digestive system were primarily treated by Acacia seyal, Bridelia micrantha, Ficus vasta, Maytenus heterophylla, Myrica salicifolia, and Verbena officinalis (ROP $=100 \%$ ). Respiratory system diseases were mainly cured by Catha edulis, Ocimum lamiifolium, and Pittosporum viridiflorum $(\mathrm{ROP}=$ $100 \%)$. The genitourinary ailments were cured using Foeniculum vulgare (ROP $=95 \%)$ and Lepidium sativum (ROP $=88 \%)$; diseases of the musculoskeletal system by Ajuga integrifolia $(R O P=100 \%)$; diseases of the skin and subcutaneous tissue by Argemone mexicana, Plantago lanceolata and Salvia nilotica $(\mathrm{ROP}=100 \%)$; rabies is mostly treated by Phytolacca dodecandra $(\mathrm{ROP}=73 \%)$; Carica papaya (ROP $=100 \%)$ was used to cure malaria; For the category of dental and oral problems Ekebergia capensis and Datura stramonium (ROP $=100 \%$ ) were found to be the most important. Liver complaints were mainly treated by Ensete ventricosum $(\mathrm{ROP}=84 \%)$, Justicia schimperiana $(\mathrm{ROP}=100 \%)$ and a fungus sp. Calvatia sp. (Agaricaceae) (ROP $=100 \%)$; inflammations related to anthrax were mostly treated by Brassica nigra $(\mathrm{ROP}=100 \%)$ and Polygala sadebeckiana $(\mathrm{ROP}=84 \%)$.

\section{Similarity among the community based on all cited medicinal plants}

Computed dissimilarity coefficient using all cited medicinal plants was above 0.5 (Table 1 ). In relative term, Mareqo and Muhir-Aklil showed the smallest similarity $(\mathrm{JI}=0.26)$ and highest similarity $(\mathrm{JI}=0.47)$ was obtained between Silti and Wulbareg districts. 
High cophenetic correlation coefficient (0.92) was obtained using the UPGMA (average) clustering method, unlike the single, complete and ward methods that had cophenetic correlation coefficient of 0.90 , 0.88 , and 0.73 , respectively. High cophenetic correlation coefficient indicates that the resulting dendrogram is a good fit of the reality. The dendrogram formed, using UPGMA (average) clustering method clustered the study sites broadly into two groups that are fairly close (Figure 3 ). In the first cluster A1, grouped Meskan, Sodo, Cheha, and Muhir-Aklil as deemed most similar. In Cluster A2, Qebena, Mareko, Silti and Wulbareg were grouped as similar or closely related. The first two sites in cluster A2 (Qebena and Mareqo) were outside the sub-cluster formed between Silti and Wulbareg.

\section{Similarity among the community based on culturally most important medicinal plants ( $R O P \geq 50 \%)$.}

Similarity coefficient between study communities based on culturally most important medicinal plants range between 0.11-0.45 (Table 2). In relative term, the most dissimilar study sites were Muhir-Aklil and Wulbareg $(\mathrm{JI}=0.10)$. Highest similarity was obtained between Cheha and Qebena districts $(\mathrm{JI}=0.45)$.

Cophenetic correlation coefficient obtained using UPGMA, Complete, Ward and Single methods were 0.78, $0.77,0.74$ and 0.71 , respectively. The dendrogram obtained from the UPGMA (average) clustering method grouped the study districts into two clusters but differently from the dendrogram obtained using all cited plant species (Figure 4). In cluster B1, Cheha and Qebena are grouped into the same sub-cluster while Muhir-Aklil stands alone on the same branch. On the other hand, in cluster B2 Meskan and Sodo were clustered together, also Silti and Mareqo on the same branch with Wulbareg as an out-group.

\section{Marketability of medicinal plants}

Few medicinal plants were being sold in six open markets visited. These medicinal plants are commonly used and well known by the local peoples. The medicinal plants encountered in the market places were sold or bought for medicinal and non-medicinal uses. Across the study districts, six herbs (Artemisia afra, Hagenia abyssinica, Lepidium sativum, Polygala sadebeckiana, Satureja abyssinica, and Silene macrosolen) were solely sold for their medicinal values and used as a source of income (Table 3). Thirteen plants (Allium sativum, Brassica carinata, Brassica nigra, Capsicum annuum, Carica papaya, Citrus aurantifolius, Coffea arabica, Cucurbita pepo, Lycopersicum esculantum, Ocimum basilicum, Rhamnus prinoides, Ruta chalepensis and Zingiber officinale) were sold at local markets primarily for their non-medicinal uses. All of the medicinal sellers encountered were women. The women's pointed out that, they collect the plant/ plant parts either from their own garden, purchased from medicinal plant sellers or collected from nearby forest patches in the zones. For example, Silene macrosolen which is reported to grow mainly in the highlands of Meskan District is sold to the sellers in Agana (near to cheha), Butajira (Meskan) and Bui (Sodo) open markets. Satureja abyssinica also grows in highland areas of Cheha and Meskan districts and the people of Silti district procure the plant from Qebet open market found in the area. With regard to MPs trading, a handful or a cup of medicinal plant parts (leaves or seeds) cost a minimum price exchange of 5 birr (0.14\$). An informant in Mareqo district reported that, a 
practice of cultivating and trading half a kilo of Jatropha curcas seeds to a neighboring healer living in Silti District worth 20 birr (0.6\$).

\section{Medicinal plants naming - Ethnotaxonomy}

Nomenclature of medicinal plant sometimes involve meaning related to the plant use or other suggestive information of the plant. This was revealed in the local names of 23 medicinal plant species; 4 of the local names reflect medicinal uses and the remaining 19 species indicate morphological character (growth form, truck color and leaf shape), poisonous, test and smell of the plants (Table 4). Seventy-nine percent of the medicinal plant species have local names in one or more local languages of the studied districts that are also sometimes used similarly or with a little differed intonation among the communities. In a few cases one local name is used for many species that have similar medicinal use. For example, local name 'Kureshe' was used for Crinum abyssinicum, Sauromatum venosum, and Tacca leontopetaloides which are used to cure livestock ailments (anthrax and blackleg).

\section{Discussion}

\section{Relative healing potential of medicinal plants: Rank order priority (ROP)}

High ROP value indicates good healing potential of a plant and the tendency of informants relying on specific medicinal plant species for treating the respective diseases reported. In the present study, the results showed that the number of plants (80 plant species, 33\%) that score ROP value greater than $50 \%$ is considerably smaller than that of plants with ROP $<50 \%$ (164 plant species, $67 \%$ ), despite the fact that these plants are with more frequent uses. This may be probably due to the diminishing popularity of many of the herbal medicine used among the study groups as stated in Friedman et al. [35]. It is also probable that the peoples are becoming selective and only use plants that are accepted in the culture as being more effective. Plants with computed ROP value at least $50 \%$ are referred here as culturally important medicinal plant species. Of 80 culturally important plant species, 37 species attained ROP values equals to $100 \%$ and might be taken as highly regarded and widely used in the community. These plants are widely known in the community and believed to be more effective [40,41]. Medicinal plant species with ROP $=100 \%$ in different study sites includes, Acacia seyal, Bridelia micrantha, Ficus vasta, Maytenus heterophylla, Myrica salicifolia, and Verbena officinalis used to treat indigestion (Qebena, Wulbarg, Muhir-Aklil and Meskan); Ajuga integrifolia used to treating abdominal pain (Sodo, Qebena, Wulbareg ), Datura stramonium and Ekebergia capensis used to treat toothache (Meskan, Wulbareg); Prunus persica and Withania somnifera to treat general malaise (Michi) (Cheha, Meskan, Muhir-Aklil); Plectranthus minutiflorus to treat after pains (Wulbareg); Calvatia sp. to treat liver problems (Silti, Wulbareg); and Carica papaya to treat Malaria (Silti, Qebena). The high FL for these species indicated the outstanding choice of informants for treating specific illness [41]. High FL also indicates the similarity of use reports for a given species whereas low FL are obtained for plant species that are used for many different purposes [40]. In these use categories, high number of medicinal plants is used in the treatment of abdominal pain. This could suggest the high prevalence of the disease in the study districts. This 
might also indicate that peoples usually use more plants (communicate the knowledge) in order to cure frequently occurring ailments. In deed this support the mere fact that a problem leads to a solution.

\section{Cross-cultural view of medicinal plants use similarity among the study groups}

Computed dissimilarity coefficient using all cited medicinal plants was above 0.5 , reflecting the fact that co-presence of plant species cited were less than $50 \%$. By using this value, the clustering displayed the similarities between the studies sites as mostly represented in the terms of ethnicity. This indicated that the same ethnic group generally shared many medicinal plants. Ethnic groups are defined as relating to large groups of people classed according to common racial, tribal, religious, linguistic, or cultural origin or background [42]. This historical linkage in the study communities is shown in the vestiges of mutual influence in medicinal plant application in one or the other way. This was seen in the first dendrogram (Figure 3), in which districts from same ethnic groups were grouped together. Cheha, Muhir-Aklil, Meskan, and Sodo from Gurage ethnic groups, Silti and Wulbareg from Silti ethnic group, Qebena and Mareqo districts stands alone and representing Qebena and Mareqo ethnic group respectively. Several studies have emphasized that ethnic, social, cultural and geographical factors as the main controllers of the number of species used by communities $[13,43,44]$. In other way, as indicated in previous studies plant use knowledge can passed down from generation to generation vertically after being taught by a family member (ancestral knowledge) or horizontally by sharing of information between peers [45-47]. One can assume from the present study that most of the free listed plants knowledge have been passing from their ancestors. In this case the differences obtained in all listed plants known by the community in the respective districts could be resulted from the route of knowledge transfer or it has ethnic origin touch.

Dendrogram obtained based on culturally important plants is completely different from the one based on all cited plants. Unlike the first it ignores ethnic reflection. Thus, pattern of distribution of culturally important medicinal plant species (plants that scored ROP $\geq 50 \%$ ) across the respective study sites could provide grounds to make further points for consideration and to infer about the way in which these plant species are being used in the community. In the cluster B1 (Figure 4), Cheha and Qebena are grouped into the same sub-cluster while Muhir-Aklil stands alone on the same branch. Whereas, cluster B2 clustered Meskan and Sodo districts together, and also Silti and Mareqo districts on the same branch with Wulbareg as an out-group. Here, it could be suggested that other factors rather than ethnical background as to explain the difference in clustering. Factors such as close vicinity and geographical orientation of the respected study sites seems to have an important influence on culturally important medicinal plant species. The clustering result suggested that groups that are geographically close often exchange information on most important plants. This would mean that with time, communities living in an area irrespective of their ethnicity, exchange knowledge that is vital for survival. From the second cluster, the plant knowledge usually shared are actively used, believed to be tested through time and considered effective in the community. The influence of proximity is future explain by informants in which communities in Wulbareg district have a long historical relationship with communities of Alaba district (a different ethnic group not included in the present study) and lived in close proximity. Consequently, the fact that Wulbareg stands as an out-group in the second cluster might be supposed that communities in 
Wulbareg exchange medicinal plant use knowledge with the people of Alaba district living in close proximity than their relatives in Silti. Geographical proximity of communities as the most influential factor of similarities in the use of medicinal plant species was also stated in the findings of Coe [48] and Avocèvou-Ayisso et al. [9]. In general, different studies ascertain that local availability of plant species, specific environmental condition, and indigenous knowledge of a particular ethnic group mainly determine the medicinal plant lore of a community $[49,50]$. Through time, however, knowledge exchange that can occur through friendships, kinship, inter-ethnical marriages and togetherness alter medicinal plant lore of a community [51]. This fact is recognized by the informants in which groups live in nearby areas have mutual attraction than with areas relatively far irrespective of their ethnic belongingness.

\section{Nomenclature of medicinal plants -Ethnotaxonomy}

Local languages are essential for transferring locally specific traditional knowledge that is vital for conserving the local environment and respective uses [52]. Local plant names occasionally had information for understanding their use or other property of the plants. This was reflected in the present study as some plants (4 plant species) were named after the ailment which the plants are used against and few more names (19 plant species) indicate the morphology (growth form, truck color and leaf shape), being poisonous, or test and odor. Naming system using the disease treated and colors was similarly reported in an ethnobotanical study of Kafficho, Ethiopia by Awas and Demissew, [6].

\section{Conclusions}

Existing patterns of medicinal plant use vary among the studied districts as a function of combined effect of socio-cultural acceptance (through exchange of information), geographic proximity between groups, and market access. As a result, in spite of medicinal plants availability in the locality, plants that are widely accepted in the culture have higher ROP values, best preferred and are sold in the local markets where most can have easy access to find. Culturally most important plants are widely used and best shared with nearby communities and it could imply current (new) knowledge being practiced in the community. Unlike plants which are less frequently used by the community, in which the plant use knowledge is conserved in the society (same ethnic group) and mostly not shared. Therefore this study recommends undertaking detailed studies across different cultures and identification of mostly shared and preferred medicinal plants for further phytochemical and pharmacological research.

\section{Declarations}

\section{Ethics approval and consent to participate}

Written permission to conduct the research was obtained from the respective zone and district administrative officials. Prior informed consent was obtained from each informant before every interview.

\section{Consent for publication}


Not applicable

\section{Availability of data and materials}

All data generated or analysed during this study are included in this published article and its supplementary information files are attached as Additional file 1, 2 \& 3.

\section{Funding}

Not applicable

\section{Competing interests}

The authors declare that they have no competing interests.

\section{Authors' contributions}

All authors have equal contribution for this work and all have read and approved the final

manuscript.

\section{Acknowledgments}

We are highly indebted to all informants who generously shared their knowledge on medicinal plants and for their hospitality; to administrative officials of the districts for their cooperation during field study. We would also like to thank the Department of Plant Biology and Biodiversity Management \& the Thematic Research Grant, Addis Ababa University; and University of Gondar for financial support. Staff members of The National Herbarium (Ethiopia) are acknowledged for their cooperation at the herbarium work. We are also grateful to Mesfin Sahile for developing the digital maps of the study area.

\section{Author information}

${ }^{1}$ Department of Biology, College of Natural and Computational Sciences, Kotebe Metropolitan University, P.O. Box 31248, Addis Ababa, Ethiopia

${ }^{2}$ Department of Plant Biology and Biodiversity Management, College of Natural

Sciences, Addis Ababa University, P.O. Box 3434, Addis Ababa, Ethiopia

${ }^{3}$ Laboratory for Tropical and Subtropical Agriculture and Ethnobotany, Department of Plant Production, Faculty of Bio-Science Engineering, Ghent University, Coupure links 653, 9000 Gent, Belgium 
${ }^{4}$ Faculty of Tropical AgriSciences, Czech University of Life Sciences Prague, Kamycka 129, Prague 6 Suchdol, 165 21, Czech Republic

\section{References}

1. Nwauzoma AB, Dappa MS: Ethnobotanical Studies of Port Harcourt Metropolis, Nigeria. ISRN Botany 2013, 1-11.

2. Smallacombe S, Davis M, Quiggin R: Scoping Project on Aboriginal Traditional Knowledge. Desert Knowledge Cooperative Research Centre, Alice Springs; 2006.

3. Timbrook, J: Ethnobiotica.Journal of Ethnobiology 2004, 24(2):5-6.

4. Reyes-García V, Marti N, McDade TW, Tanner S, Vadez V: Concepts and methods in studies measuring individual ethnobotanical knowledge.Journal of Ethnobiology 2007, 27:108-203.

5. http://www.livescience.com/21478-what-is-culture-definition-of-culture.html. accessed on 18 Nov 2019.

6. Awas T, Demissew S: Ethnobotanical study of medicinal plants in Kafficho people, Southwestern Ethiopia. In: Proceedings of the 16th International Conference of Ethiopian Studies. Edited by Birhanu, T. and B. Shiferaw. Addis Ababa, Ethiopia; 2009.

7. Payyappallimana U: Role of Traditional Medicine in Primary Healthcare: An Overview of Perspectives and Challenges. Yokohama Journal of Social Sciences 2010, 14(6): 57-77.

8. Bekele E: Study on Actual Situation of Medicinal Plants in Ethiopia; 2007 http://www.endashaw.com

9. Avocèvou-Ayisso C, Avohou TH, Oumorou M, Dossou G, Sinsin B: Ethnobotany of Pentadesma butyracea in Benin: A quantitative approach. Ethnobotany Research \& Applications 2011, 9:151-166.

10. Junsongduang A, Balslev H, Inta A, Jampeetong A, Wangpakapattanawong P: Karen and Lawa medicinal plant use: Uniformity or ethnic divergence? Journal of Ethnopharmacology 2014, 151:517527.

11. Pardo-De-Santayana M, Macia MJ: Biodiversity: The benefits of traditional knowledge.Nature 2015, 518:487-488.

12. Abebe D, Ayehu A: Medicinal plants and Enigmatic Health practices of Northern Ethiopia. Addis Ababa, Ethiopia: B.S.P.E.; 1993.

13. Ayantunde AA, Briejer M, Hiernaux $P$, Udo HMJ, Tabo R: Botanical knowledge and its differentiation by age, gender and ethnicity in Southwestern Niger.Human Ecology 2008, 36:881-889.

14. Pieroni A: Medicinal plants and food medicines in the folk traditions of the upper Lucca Province, Italy.Journal of Ethnopharmacol 2000, 70: 235-73.

15. Guarrera PM, Lucia LM: Ethnobotanical remarks on central and southern Italy. Journal of Ethnobiology and Ethnomedicine 2007, 3(23).

16. Kuria MW, Njenga PK, Ngumi VW: Ethnobotanical studies of Strychnos henningsii in five (Gilg.) natural habitats in Kenya.International Journal of Plant Research 2012, 1(6):63-64. 
17. Pfeiffer JM, Butz RJ: Assessing cultural and ecological variation in ethnobiological research: the importance of gender.Journal of Ethnobiology 2005, 25(2):240-278.

18. Kidane B, Andel T, Josephus L, Asfaw Z: Use and management of traditional medicinal plants by Maale and Ari ethnic communities in southern Ethiopia.Journal of Ethnobiology and Ethnomedicine 2014, 10(46):1-15.

19. GZFEDD (Gurage Zone Finance and Economy Development Department): The Gurage Zone five year (2010-2014) Growth and Transformation plan (Amharic version) Wolkite, Ethiopia; 2010.

20. Nishi M: Making and unmaking of the nation-state and ethnicity in modern Ethiopia: a study on the history of the Silte people.African Study Monographs 2005, 29:157-168.

21. Shack W: The Gurage: A people of the Ensete Culture. London, Oxford University Press; 1966.

22. Friis I, Demissew S, Van Breugel P: Atlas of the Potential Vegetation of Ethiopia. Addis Ababa University Press and Shama Books; 2011.

23. Gomez-Beloz A: Plant use knowledge of the Winikina Warao: The case for questionnaires in ethnobotany. Economy Botany 2002, 56:231-241.

24. Cotton CM: Ethnobotany: Principles and Applications. John Wiley and Sons, New York; 1996.

25. Bernard HR: Research Methods in Anthropology: Qualitative and quantitative methods. $3^{\text {rd }}$ Alta Mira Press ,Walnut Creek, California 2002, 141-162.

26. Van Damme P, Kindt R: Ethnobotanical methods. In: Agroforestry tree domestication: a primer, Dawson, I., Harwood, C., Jamnadass, R. and Beniest, J. (eds.). The World Agroforestry Centre, Nairobi, Kenya 2012, 28-35.

27. Edwards S, Demissew S, Hedberg I, editors. Flora of Ethiopia and Eritrea. Hydrocharitaceae to Arecaceae volume 6. Ethiopia: Department of Systematic Botany, Uppsala University, Uppsala and The National Herbarium, Addis Ababa University, Addis Ababa; 1997.

28. Edwards S, Tadesse M, Demissew S, Hedberg I, editors. Flora of Ethiopia and Eritrea. Magnoliaceae to Flacourtiaceae volume 2 part 1. Ethiopia: Department of Systematic Botany, Uppsala University, Uppsala and The National Herbarium, Addis Ababa University, Addis Ababa; 2000.

29. Edwards S, Tadesse M, Hedberg I, editors. Flora of Ethiopia and Eritrea. Canellaceae to Euphorbiaceae volume 2 part 2. Ethiopia: Department of Systematic Botany, Uppsala University, Uppsala and The National Herbarium, Addis Ababa University, Addis Ababa; 1995.

30. Hedberg I, Edwards S, editors. Flora of Ethiopia and Eritrea. Poaceae (Gramineae) volume 7. Ethiopia: Department of Systematic Botany, Uppsala University, Uppsala and The National Herbarium, Addis Ababa University, Addis Ababa; 1995.

31. Hedberg I, Edwards S, editors. Flora of Ethiopia and Eritrea. Pittosporaceae to Araliaceae volume 3. Ethiopia: Department of Systematic Botany, Uppsala University, Uppsala and The National Herbarium, Addis Ababa University, Addis Ababa; 1989.

32. Hedberg I, Edwards S, Nemomissa S, editors. Flora of Ethiopia and Eritrea. Apiaceae to Dipsacaceae volume 4 part 1. Ethiopia: Department of Systematic Botany, Uppsala University, Uppsala and the 
National Herbarium, Addis Ababa University, Addis Ababa; 2003.

33. Hedberg I, Friis I, Edwards S, editors. Flora of Ethiopia and Eritrea. Asteraceae volume 4 part 2. Ethiopia: Department of Systematic Botany, Uppsala University, Uppsala and the National Herbarium, Addis Ababa University, Addis Ababa; 2004.

34. Hedberg I, Kelbessa E, Edwards S, Demissew S, Persson E, editors. Flora of Ethiopia and Eritrea. Plantaginaceae volume 5. Ethiopia: Department of Systematic Botany, Uppsala University, Uppsala and The National Herbarium, Addis Ababa University, Addis Ababa; 2006.

35. Friedman J, Yaniv Z, Dafni A, Palevitch D: A preliminary classification of the healing potential of medicinal plants, based on a rational analysis of an ethnopharmacological field survey among Bedouins in the Negev desert, Israel. Journal of Ethnopharmacology 1986, 16: 275-278.

36. Musa MS, Abdelrasool FE, Elsheikh EA, Ahmed L, Mahmoud A, Yagi SM: Ethnobotanical study of medicinal plants in the Blue Nile State, South-eastern Sudan.Journal of Medicinal Plants Research 2011, 5(17):4287-97.

37. Al-Quran S: Taxonomical and Pharmacological Survey of Therapeutic Plants in Jordan.Journal of Natural Products 2008, 1:10-26.

38. Borcard D, Gillet F, Legendre P: Numerical Ecology with R. New York. Springer; 2011.

39. Höft M, Barik SK, Lykke AM: Quantitative Ethnobotany: Application of Multivariate and Statistical Analyses in Ethnobotany.People and Plants working paper 6. UNESCO, Paris; 1999.

40. Enyew A, Asfaw Z, Kelbessa E, Nagappan R: Ethnobotanical Study of Traditional Medicinal Plants in and Around Fiche District, Central Ethiopia. Current Research Journal of Biological Sciences 2014, 6(4):154-167.

41. Baydoun S, Lamis C, Helenaa D, Nellya A: Ethnopharmacological survey of medicinal plants used in traditional medicine by the communities of Mount Hermon, Lebanon.Journal of Ethnopharmacology 2015, 173:139-156.

42. merriam-webster.com/dictionary/ethnic accessed on 18 Nov 2019.

43. Vandebroek I, Calewaert JB, De Jonckheere S, Sanca S, Semo L, Van Damme P, Puyvelde LV, de Kimpe N: Use of medicinal plants and pharmaceuticals by indigenous communities in the Bolivian Andes and Amazon.Bulletin of World Health Organization 2004, 82(4):243-250.

44. Sop TK, Oldeland J, Bognounou F, Schmiedel U. Thiombiano A: Ethnobotanical knowledge and valuation of woody plants species: a comparative analysis of three ethnic groups from the sub-Sahel of Burkina Faso.Environment Development and Sustainability 2012, 14:627-649.

45. Gessler MC, Msuya DE, Nkunya MHH, Mwasumbi LB, Schar A, Heinrich M. Tanner M: Traditional healers in Tanzania: The treatment of malaria with plant remedies. Journal of Ethnopharmacoly 1995, 48:131-144.

46. Ndubani P, Höjer B: Traditional healers and the treatment of sexually transmitted illnesses in rural Zambia.Journal of Ethnopharmacology 1999, 67:15-25. 
47. Setalaphruk C, Price LL: Children's traditional ecological knowledge of wild food resources: a case study in a rural village in Northeast. Thailand Journal of Ethnobiology and Ethnomedicine 2007, 3:33 doi:10.1186/1746-4269-3-33

48. Coe FG: Ethnobotany of the Rama of Southeastern Nicaragua and comparisons with Miskitu plant lore.Economy Botany 2008, 62(1):40-59.

49. Cunningham AB: Applied ethnobotany: people, wild plant use and conservation. Earthscan, London, UK; 2001.

50. Gouwakinnou GN, Lykke AM, Assogbadjo AE, Sinsin B: Local knowledge, pattern and diversity of use of Sclerocarya birrea.Journal of Ethnobiology and Ethnomedicine 2011, 7: 8.

51. Menendez-Baceta G, Aceituno-Mata L, Reyes-García V, Tardío J, Salpeteur M, Pardo-de-Santayana M: The importance of cultural factors in the distribution of medicinal plant knowledge: A case study in four Basque regions.Journal of Ethnopharmacology 2015, 161:116-127.

52. Ross, S: Talking the Walk: Language as the missing ingredient of biodiversity conservation? An investigation of plant knowledge in the West Usambara Mountains, Tanzania. School of Development Studies, University of East Anglia;

\section{Tables}

Table 1. Similarity of medicinal plant species cited among the study districts

\begin{tabular}{lllllllll}
\hline & Sodo & $\begin{array}{l}\text { Muhir- } \\
\text { Aklil }\end{array}$ & Cheha & Silti & Wulbareg Qebena Mareqo Meskan \\
\hline Sodo & 1 & & & & & & & \\
Muhir-Aklil & 0.40 & 1 & & & & & & \\
Cheha & 0.39 & 0.42 & 1 & & & & & \\
Silti & 0.37 & 0.38 & 0.34 & 1 & & & & \\
Wulbareg & 0.35 & 0.27 & 0.35 & 0.47 & 1 & & & \\
Qebena & 0.31 & 0.34 & 0.38 & 0.34 & 0.37 & 1 & & \\
Mareqo & 0.35 & 0.26 & 0.30 & 0.45 & 0.41 & 0.35 & 1 & \\
Meskan & 0.37 & 0.35 & 0.37 & 0.42 & 0.34 & 0.32 & 0.34 & 1 \\
\hline
\end{tabular}

Jaccard similarity indices $(0-1), 1=$ similar; $0=$ dissimilar. Note that the matrix is symmetrical about the diagonal

Table 2. Similarity of culturally most important medicinal plant species among the study

\begin{tabular}{|c|c|c|c|c|c|c|c|c|}
\hline & Sodo & Muhir-Aklil & Cheha & Silti & \multicolumn{4}{|c|}{ WulbaregQebena Mareqo Meskan } \\
\hline Sodo & 1 & & & & & & & \\
\hline Muhir-Aklil & 0.12 & 1 & & & & & & \\
\hline Cheha & 0.11 & 0.30 & 1 & & & & & \\
\hline Silti & 0.12 & 0.19 & 0.22 & 1 & & & & \\
\hline Wulbareg & 0.15 & 0.10 & 0.20 & 0.33 & 1 & & & \\
\hline Qebena & 0.13 & 0.29 & 0.45 & 0.23 & 0.18 & 1 & & \\
\hline Mareqo & 0.14 & 0.19 & 0.25 & 0.43 & 0.24 & 0.27 & 1 & \\
\hline Meskan & 0.31 & 0.27 & 0.30 & 0.34 & 0.14 & 0.20 & 0.29 & 1 \\
\hline
\end{tabular}


Jaccard similarity indices $(0-1), 1=$ similar; $0=$ dissimilar. Note that the matrix is symmetrical about the diagonal

Table 3. Checklist of plant species used only for their medicinal values and sold in the open markets visited in the study area

\begin{tabular}{|c|c|c|c|c|c|c|c|}
\hline \multirow[b]{2}{*}{ Species } & \multicolumn{7}{|c|}{ Open markets } \\
\hline & Agena & Bui & Butajira & Hawariyat & Imdiber & Qibet & Qoshe \\
\hline Artemisia afra & $\ddot{\mathrm{O}}$ & & $\tilde{\mathrm{O}}$ & & & & Ö \\
\hline Hagenia abyssinica & $\overline{\mathrm{O}}$ & & $\ddot{\mathrm{O}}$ & & Ö & & \\
\hline Lepidium sativum & Ö & Ö & Ö & Ö & Ö & Ö & Ö \\
\hline Polygala sadebeckiana & Ö & & & Ö & Ö & & \\
\hline Silene macrosolen & Ö & $\ddot{O}$ & $\ddot{O}$ & & Ö & & \\
\hline Satureja abyssinica & $\ddot{O}$ & & $\ddot{\mathrm{O}}$ & & & & \\
\hline
\end{tabular}

Agena market - near to Cheha \& Qebena; Bui market - Sodo; Butajira market - Meskan; Hawariyat market Muhir-Aklil; Imdiber market - Cheha; Qibet market - Silti; Qoshe market -Mareqo

Table 4. Local names of medicinal plants and their meaning (S- Silti; G-Guragigna; MMareqo; Q-Qebena) 


\begin{tabular}{|c|c|c|c|c|}
\hline No. & Scientific name & Local name & $\begin{array}{l}\text { Direct meaning of the local name } \\
\text { in English }\end{array}$ & $\begin{array}{l}\text { Meaning } \\
\text { reflecting }\end{array}$ \\
\hline 1 & Acacia abyssinica [Fabaceae] & $\begin{array}{l}\text { Teme-gerar (S, } \\
\text { G) }\end{array}$ & Black acacia & Trunk color \\
\hline 2 & Acacia seyal [Fabaceae] & $\begin{array}{l}\text { Wacho-gerar (S, } \\
\text { G) }\end{array}$ & Red acacia & Trunk color \\
\hline 3 & Ajuga integrifolia [Lamiaceae] & \begin{tabular}{|l|} 
Anamuro, ema \\
telit (G)
\end{tabular} & $\begin{array}{l}\text { Makes an infant hate breast } \\
\text { feeding, used purposfuly to stop } \\
\text { breast feeding }\end{array}$ & Bitter test \\
\hline 4 & Aloe pubescens [Aloaceae] & Merdedeye (G) & \begin{tabular}{|l}
$\begin{array}{l}\text { Saw like (a tool with toothed } \\
\text { blade) }\end{array}$ \\
\end{tabular} & $\begin{array}{l}\text { Marginal teeth of } \\
\text { the leaf }\end{array}$ \\
\hline 5 & \begin{tabular}{|l} 
Artemisia \\
abyssinica [Asteraceae]
\end{tabular} & Chiyanchiye (G) & Bad smell & $\begin{array}{l}\text { The leaves has } \\
\text { bad smell }\end{array}$ \\
\hline 6 & \begin{tabular}{|l|} 
Brucea \\
antidysenterica [Simaroubaceae]
\end{tabular} & $\begin{array}{l}\begin{array}{l}\text { Yemoyet bosha } \\
\text { (G) }\end{array} \\
\end{array}$ & $\begin{array}{l}\text { Leaves of moyet (a social group } \\
\text { known to exist in Gurage) that is } \\
\text { used during cultural ceremony }\end{array}$ & Ceremonial use \\
\hline 7 & $\begin{array}{l}\begin{array}{l}\text { Clematis } \\
\text { simensis [Ranunculaceae] }\end{array} \\
\end{array}$ & $\begin{array}{l}\begin{array}{l}\text { Yegawa wedero } \\
\text { (G) }\end{array} \\
\end{array}$ & Fool's rope & $\begin{array}{l}\text { Climber growth } \\
\text { form }\end{array}$ \\
\hline 8 & \begin{tabular}{|l} 
Convolvulus \\
sagittatus [Convolvulaceae]
\end{tabular} & Minen debo (M) & Medicinal & Medicinal use \\
\hline 9 & Crotalaria incana [Fabaceae] & Meza qutel (G) & Leave for wound & Medicinal use \\
\hline 10 & $\begin{array}{l}\text { Cucumis } \\
\text { ficifolius [Cucurbitaceae] }\end{array}$ & \begin{tabular}{l}
\multicolumn{2}{l}{ Hulgerecho } \\
$(\mathrm{M}), \quad$ Adene \\
debaqula (Q), \\
Yemeder \\
qimbiba, Yafer- \\
granger (G), \\
Yale-tay (S) \\
\end{tabular} & $\begin{array}{l}\text { Fruits laied on land (G), Monkey's } \\
\text { genital organ (Q) }\end{array}$ & $\begin{array}{l}\text { Fruit growth habit } \\
\text { and shape }\end{array}$ \\
\hline 11 & $\begin{array}{l}\text { Cynoglossum } \\
\text { coeruleum [Boraginaceae] }\end{array}$ & \begin{tabular}{ll|} 
Yitebtiye & $(\mathrm{G})$ \\
Bertetusa & $(\mathrm{Q})$, \\
Hatemaqo & $(\mathrm{Q}$, \\
$\mathrm{S})$, & \\
\end{tabular} & Sticky & $\begin{array}{l}\text { sticky nature of } \\
\text { the fruits }\end{array}$ \\
\hline 12 & $\begin{array}{l}\text { Cyphostemma } \\
\text { niveum [Vitaceae] }\end{array}$ & Yeseb eje (G) & Hand & Leaf shape \\
\hline 13 & Foeniculum vulgare [Apiaceae] & Wet-ambo (G) & & $\begin{array}{l}\text { Stems hollow } \\
\text { when mature }\end{array}$ \\
\hline 14 & Fuerstia africana [Lamiaceae] & $\begin{array}{l}\text { Yegiye insosla } \\
\text { (G), Nazoli (S), } \\
\text { Hureda (M) }\end{array}$ & $\begin{array}{l}\text { Insosla (Impatiens tinctoria) dark } \\
\text { red dye extracted from the tubers } \\
\text { used as beauty treatment, } \\
\text { Fuerstia africana is called for } \\
\text { dog's which is not similar to above } \\
\text { mentioned species (G) }\end{array}$ & $\begin{array}{l}\text { Color of the red } \\
\text { juice squeezed } \\
\text { from the leaves }\end{array}$ \\
\hline 15 & \begin{tabular}{|l} 
Haplocarpha \\
schimperi [Asteraceae]
\end{tabular} & \begin{tabular}{|l|}
$\begin{array}{l}\text { Ayene } \\
\text { (G) }\end{array}$ \\
\end{tabular} & Has blinding effect & $\begin{array}{l}\text { Poisonous to the } \\
\text { eyes }\end{array}$ \\
\hline 16 & Pavonia urens [Malvaceae] & Menatef (G) & \begin{tabular}{|l|} 
Trigger vomiting when feeling sick \\
\end{tabular} & Medicinal use \\
\hline
\end{tabular}




\begin{tabular}{|c|c|c|c|c|}
\hline No. & Scientific name & Local name & $\begin{array}{l}\text { Direct meaning of the local name } \\
\text { in English }\end{array}$ & $\begin{array}{l}\text { Meaning } \\
\text { reflecting }\end{array}$ \\
\hline 17 & $\begin{array}{l}\text { Polygala } \\
\text { sadebeckiana [Polygalaceae] }\end{array}$ & \begin{tabular}{|l|} 
Shime-itere \\
chiza $(\mathrm{G})$, Shime \\
yeter zebo (Q), \\
Qiteriye $(\mathrm{G})$
\end{tabular} & $\begin{array}{l}\text { Local name of antrax/blackleg (G, } \\
\text { Q); Finger like (G) }\end{array}$ & $\begin{array}{l}\text { Medicinal use, } \\
\text { root structure }\end{array}$ \\
\hline 18 & $\begin{array}{l}\text { Plantago } \\
\text { lanceolate [Plantaginaceae] }\end{array}$ & Yefur enzir (G) & Rat's ear & Leaf shape \\
\hline 19 & Rhoicissus tridentate [Vitaceae] & $\begin{array}{l}\text { Yegawa wedero } \\
\text { (G), dubi fizuta } \\
(\mathrm{Q})\end{array}$ & Fool's rope & Climbing nature \\
\hline 20 & Rhynchosia minima [Fabaceae] & Yefur enzir (G) & Rat's era & Leaf shape \\
\hline 21 & $\begin{array}{l}\text { Thunbergia } \\
\text { ruspolii [Acanthaceae] }\end{array}$ & $\begin{array}{l}\text { Yangacha } \\
\text { qomet }(\mathrm{G})\end{array}$ & Cat's Lagenaria siceraria & $\begin{array}{l}\text { Resemble fruit } \\
\text { and flower } \\
\text { (corolla) shape } \\
\text { which are smaller } \\
\text { than fruit of } \\
\text { Lagenaria } \\
\text { siceraria }\end{array}$ \\
\hline 22 & $\begin{array}{l}\text { Verbascum } \\
\text { sinaiticum [Scrophulariaceae] }\end{array}$ & $\begin{array}{|lr|}\text { Yemar } & \text { enzir } \\
(\mathrm{G}), & \text { Huleten } \\
\text { huta }(\mathrm{M}), \\
\text { Yumar amel } \\
\mathrm{S})\end{array}$ & Donkey's ear & Leaf shape \\
\hline 23 & \begin{tabular}{|l} 
Xanthium \\
strumarium [Asteraceae]
\end{tabular} & \begin{tabular}{|lr} 
Yetey- & soohe \\
$(\mathrm{G})$, & Gereba \\
uta $(\mathrm{M})$ &
\end{tabular} & Sheep's spine & $\begin{array}{l}\text { Sticky nature of } \\
\text { the fruit, usually } \\
\text { seen sticking on } \\
\text { sheep's fur }\end{array}$ \\
\hline
\end{tabular}

\section{Figures}




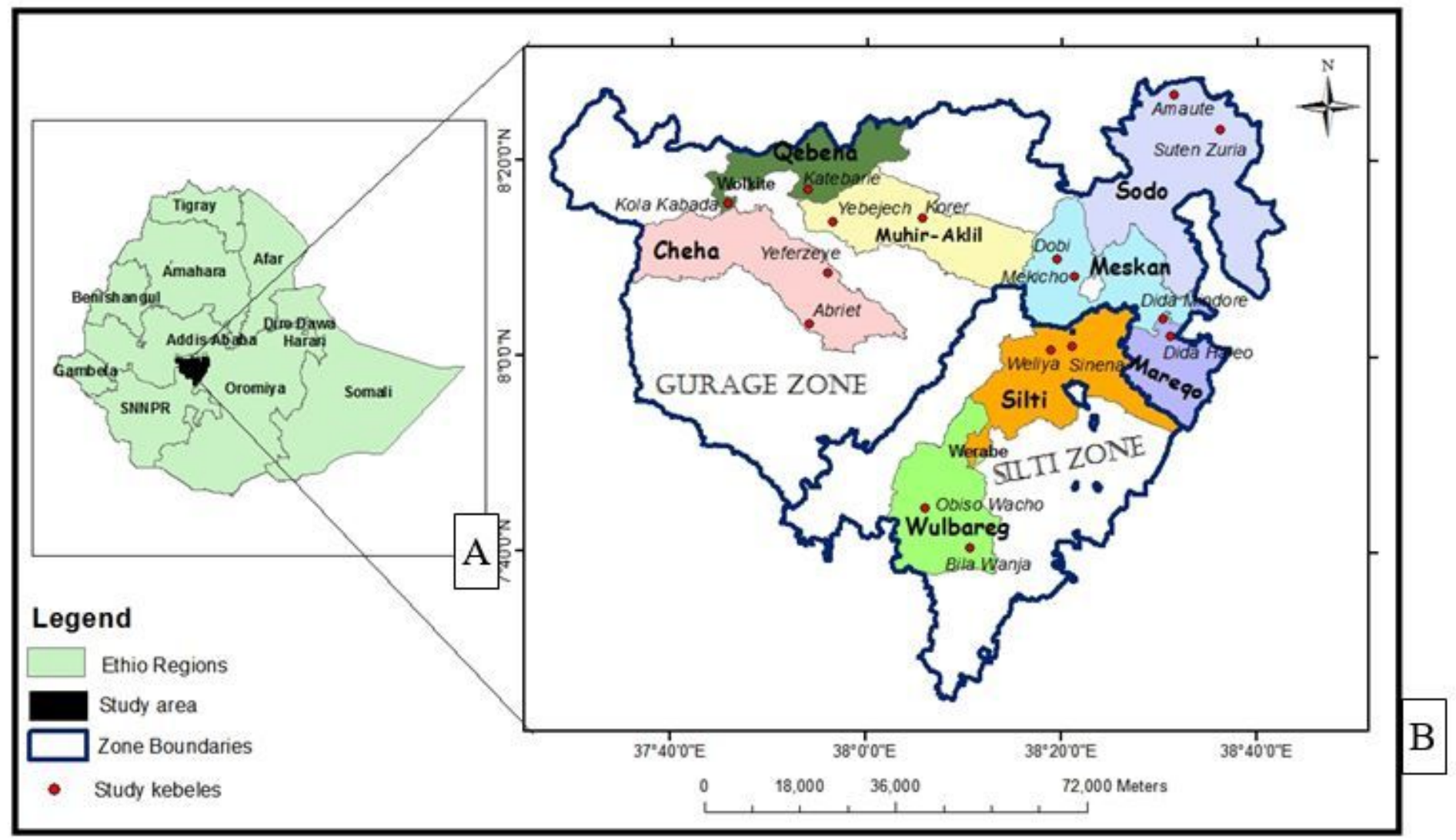

Figure 1

A). Map of Ethiopia B). The study districts in the respective zones

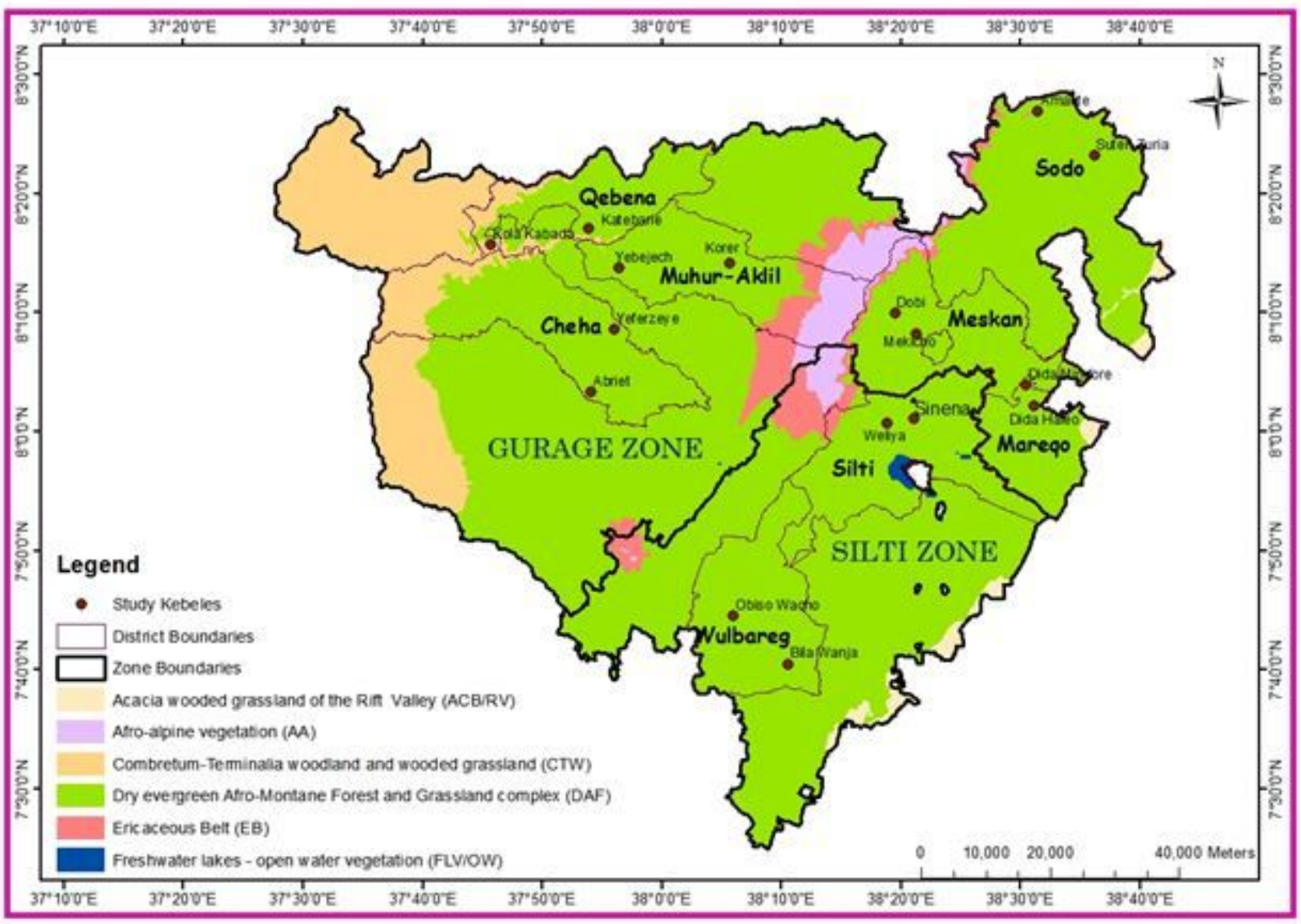


Figure 2

The vegetation types of Gurage and Silti zones (based on map in Atlas of the Potential Vegetation of Ethiopia by Friis et al. (2011))

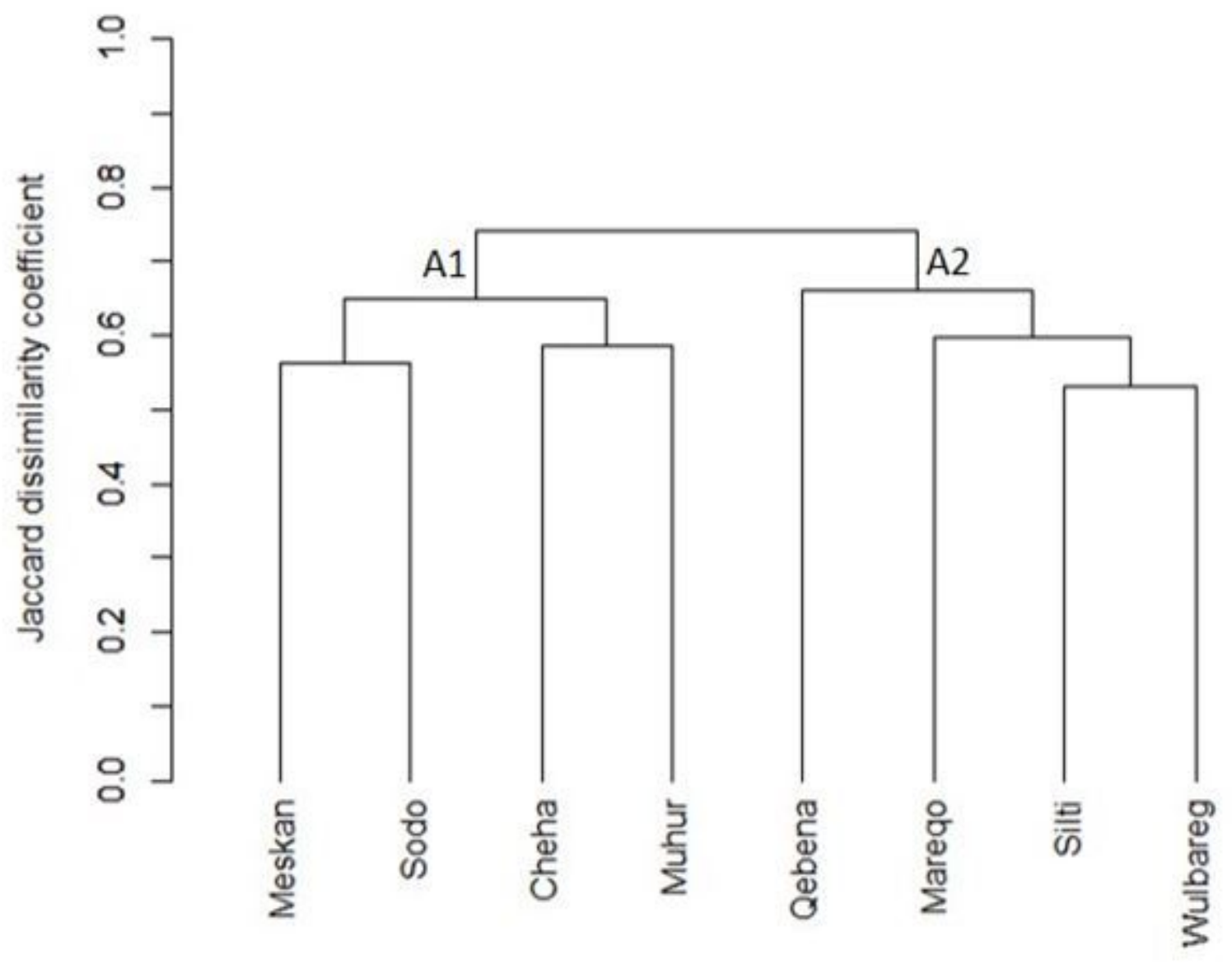

\section{Figure 3}

Dendrogram showing the dissimilarity between study districts based on all medicinal plant species mentioned (based on Jaccard dissimilarity coefficient and UPGMA clustering method) 


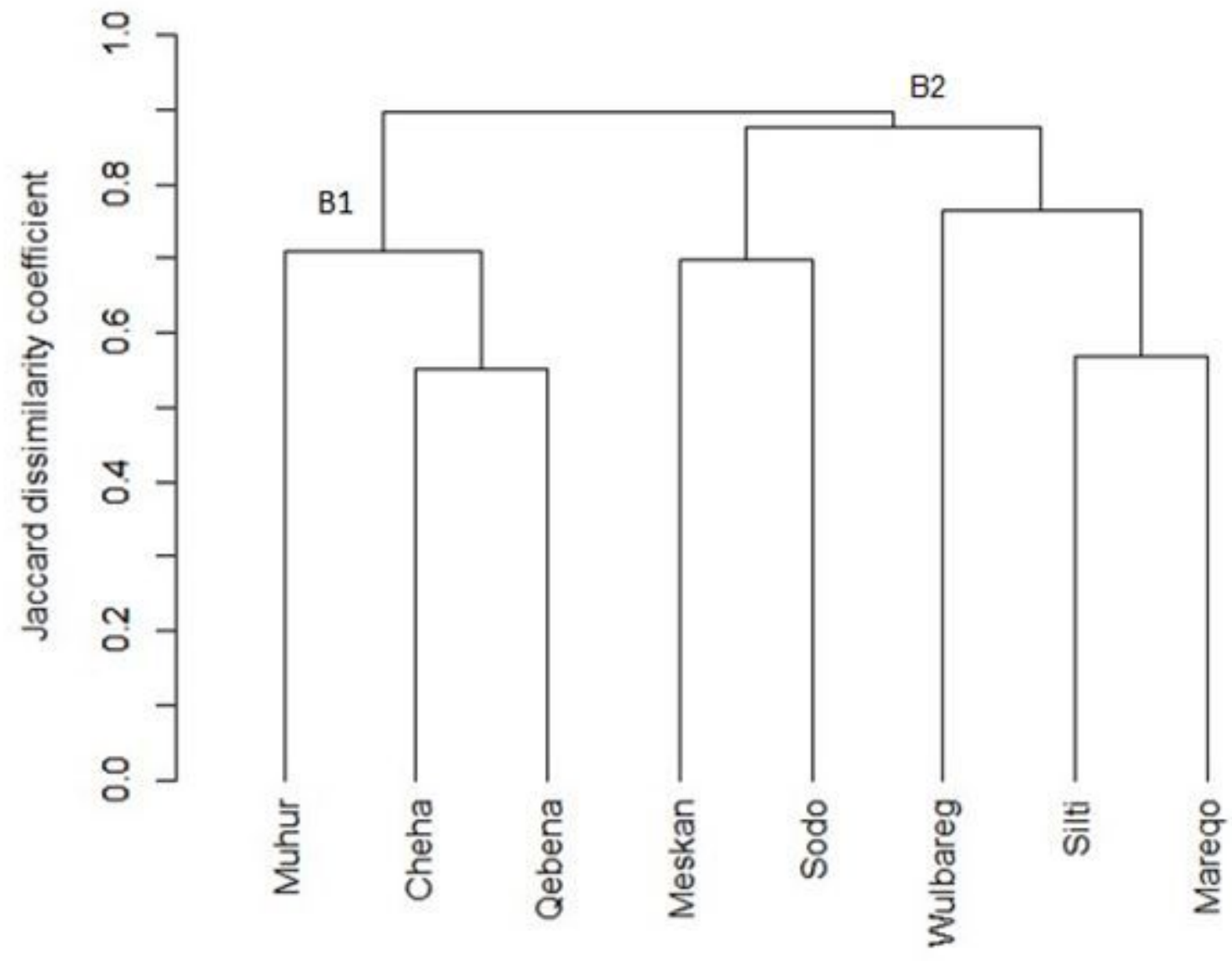

Figure 4

Dendrogram showing the dissimilarity between study sites based on important medicinal plant species (based on Jaccard dissimilarity coefficient and UPGMA clustering method)

\section{Supplementary Files}

This is a list of supplementary files associated with this preprint. Click to download.

- AdditionalmaterialsA.Teka.doc

- formula1.JPG 